\title{
Visual landmarks facilitate rodent spatial navigation in virtual reality environments
}

\author{
Isaac A. Youngstrom and Ben W. Strowbridge ${ }^{1}$ \\ Department of Neurosciences, Case Western Reserve University School of Medicine, Cleveland, Ohio 44106, USA
}

\begin{abstract}
Because many different sensory modalities contribute to spatial learning in rodents, it has been difficult to determine whether spatial navigation can be guided solely by visual cues. Rodents moving within physical environments with visual cues engage a variety of nonvisual sensory systems that cannot be easily inhibited without lesioning brain areas. Virtual reality offers a unique approach to ask whether visual landmark cues alone are sufficient to improve performance in a spatial task. We found that mice could learn to navigate between two water reward locations along a virtual bidirectional linear track using a spherical treadmill. Mice exposed to a virtual environment with vivid visual cues rendered on a single monitor increased their performance over a 3-d training regimen. Training significantly increased the percentage of time avatars controlled by the mice spent near reward locations in probe trials without water rewards. Neither improvement during training or spatial learning for reward locations occurred with mice operating a virtual environment without vivid landmarks or with mice deprived of all visual feedback. Mice operating the vivid environment developed stereotyped avatar turning behaviors when alternating between reward zones that were positively correlated with their performance on the probe trial. These results suggest that mice are able to learn to navigate to specific locations using only visual cues presented within a virtual environment rendered on a single computer monitor.
\end{abstract}

[Supplemental material is available for this article.]

Spatial memory has been long appreciated as a fundamental aspect of rodent behavior and plays an especially critical role in navigation (Morris 1981). While spatial learning has been studied extensively (Tanila et al. 1997; Knierim et al. 1998), it remains unclear how rodents utilize different types of sensory stimuli during spatial navigation and learning. Many studies have linked visual cues in the environment to spatial learning (Morris 1981; Brown et al. 2002; Janus 2004), suggesting that spatial memories can be formed using only visual information. However, visual cues can have multiple, and contradictory, effects on spatial learning. Many classic studies have emphasized the role of distant visual cues (Morris 1981). Other studies have demonstrated behavioral paradigms, where spatial memories are formed using local beacon landmarks associated with reward locations (Brown et al. 2002; Janus 2004). Surprisingly, the presence of local visual landmark cues during training can inhibit navigation ability in some paradigms (Roberts and Pearce 1999).

Spatial learning is an important process for foraging rodents, and rodents can use multiple strategies to accomplish this behavior (McDonald and White 1994). In intact rodents traveling in physical environments, self-motion generates cues that are relevant to path integration, one known mechanism of spatial navigation (for review, see Etienne and Jeffery 2004). One of these cues is head direction, which is modulated by vestibular input to headdirection cells in the anterior thalamus (Knierim et al. 1995). Lesioning the vestibular system interferes with the head-direction system and impairs spatial learning (Zheng et al. 2009; Baek et al. 2010). However, lesions of the vestibular system may have indirect effects that affect spatial function. Since no previous study has prevented horizontal vestibular activation without vestibular end organ lesions, it is not known whether the spatial memory

\footnotetext{
${ }^{1}$ Corresponding author.

E-mail bens@case.edu.

Article is online at http://www.learnmem.org/cgi/doi/10.1101/Im.023523.111.
}

impairment associated with these lesions reflects a crucial role for active, on-going vestibular input or indirect effects of the lesions. Although brain lesions have been useful in defining the requirements for different brain areas in spatial tasks (McDonald and White 1994), this approach by itself cannot address the fundamental question of whether intact animals must integrate salient input from different sensory modalities to form new spatial memories. Eliminating visual input from spatial learning paradigms is straightforward and Save and Moghaddam (1996) demonstrated that rats can navigate through a Morris water maze in the dark. However, removing all salient nonvisual input has not been feasible in nonlesioned rodents moving through physical environments. Because of these complications, it has been technically difficult to demonstrate that rodents can use only visual input to learn the spatial relationships within an environment.

One approach to address this question is to ask whether rodents can form spatial memories without moving through different physical locations. In the present study, head-fixed mice used visual stimuli presented in a virtual reality (VR) system to direct spatial navigation in the absence of relevant self-motion cues and horizontal head-motion cues. Virtual reality offers unique advantages for studying spatial navigation and can directly test whether visual cues alone are sufficient to drive learning in the absence of the appropriate nonvisual sensory input normally generated by movement through a physical environment. Recent studies used VR to demonstrate that both head-fixed mice (Harvey et al. 2009; Dombeck et al. 2010) and humans (Ekstrom et al. 2003) can develop place fields while navigating through virtual environments. Place cell activity, however, develops rapidly after rodents are exposed to novel environments (Wilson and McNaughton 1993) and remains highly plastic during training (e.g., place fields can be remapped following changes in the environment) (Lever et al. 2002). Place cell activity, therefore, cannot be directly used as a proxy for spatial learning, which develops over repeated training sessions when tested in conventional 
Morris water mazes (for review, see Morris 1984). It remains unknown whether head-fixed rodents can navigate in a virtual environment without spatial cues provided by water rewards in fixed positions. In the present study, we trained head-fixed mice to operate virtual environments by running on a levitated spherical treadmill (Fig. 1), and used behavioral tests to determine whether head-fixed mice can learn to navigate to specific locations within a VR environment. We find that visual cues are critical for mice to improve their performance in navigating between water reward locations on a linear virtual track and for improving their performance in probe trials without active rewards. These results represent the first direct demonstration of spatial learning in headfixed rodents, and suggest that visual landmark cues alone can guide spatial memory formation.

\section{Results}

We first tested whether virtual reality training improved performance on the alternating spatial reward location task. Figure $2 \mathrm{~A}$ illustrates the avatar position along a bidirectional virtual track during three bidirectional training sessions (sessions 3, 5, and 7) for one mouse. In the first bidirectional training session shown, mice spent a minority of the session moving the avatar between reward locations and tended to keep the avatar near the ends of

$\mathbf{A}_{1}$

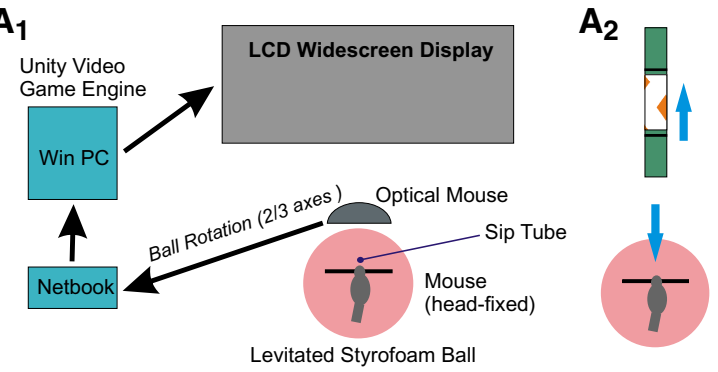

B

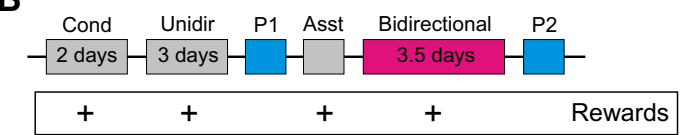

C

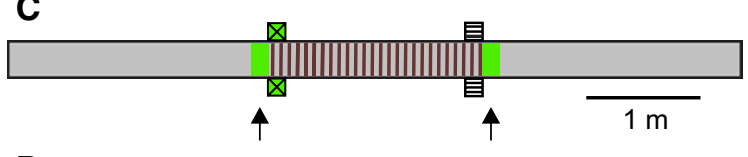

D
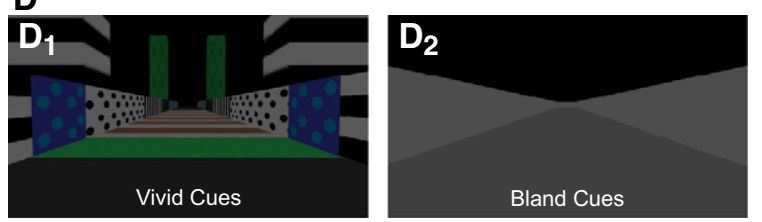

Figure 1. Head-fixed mouse virtual reality system. $\left(A_{1}\right)$ Diagram of VR system for head-fixed mice. $\left(A_{2}\right)$ Mice running on the treadmill move the VR avatar forward through the virtual environment. (B) Study plan including $2 \mathrm{~d}$ of instrument conditioning (Cond), $3 \mathrm{~d}$ of unidirectional track training (Unidir), and one bidirectional track session with assistance provided by the researcher (Asst) that were not analyzed. Results presented from unassisted bidirectional track sessions over $3.5 \mathrm{~d}$ (Bidirectional) and two probe trials without water rewards (P1 and P2). (C) Diagram of virtual bidirectional track. Water reward locations indicated by arrows. $\left(D_{1}\right)$ Example video display from a vivid track with landmark cues. $\left(D_{2}\right)$ Example display from a bland track without landmark cues. the track. Performance increased dramatically on the second day with multiple epochs, in which mice ran from one reward location to the other without contacting either end wall (e.g., middle plot in Fig. 2A,B; Supplemental Movie). This performance improvement was maintained on Day 3, and intervals between rewards became more regular (reward interval $\mathrm{CV}=0.97 \pm 0.09$ on Day 1 vs. $0.80 \pm 0.06$ on Day 3; $P<0.05$, paired $t$-test; $n=10$ animals).

In mice tested with the vivid track, the average distance traveled between rewards decreased in seven of 10 mice tested (Fig. $3 \mathrm{~A})$. The overall mean distance traveled between rewards decreased significantly with training to $69.8 \pm 5.7 \%$ of mean distance on Day 1 of training on Day $2(P<0.05 ; n=10$; paired $t$-test) and to $70.8 \pm 5.0 \%$ on Day $3(P<0.01 ; n=10)$ (Fig. 3B). Track training also significantly increased both the frequency of midzone crossings $(P<0.01$ improvement over Day 1 on Day 2, $P<0.02$ on Day 3; paired $t$-test) (Fig. 3C) and the reward frequency $(P<0.01$ on Day 2, $P<0.02$ on Day 3; paired $t$-test) (Fig. 3D). Mice exposed to bland VR environments without vivid landmark cues did not reduce the mean distance traveled between rewards (Fig. 3B) and failed to increase their performance, measured by midzone crossing frequency (gray symbols in Fig. 3C) $(P>0.05$; paired $t$-test; $n=6)$ and reward frequency (Fig. 3D) $(P>0.05)$. Similarly, mice operating with the VR engine without visual feedback ("monitor off" controls; black symbols in Fig. 3C) failed to improve their performance on all three metrics (all $P>0.05$; paired $t$-test; $n=6$ ). Mice operating the same VR system without any visual feedback (LCD monitor not powered) performed worse than either vivid or bland track group (mean distance between rewards on Day $3=16.3 \pm 3.7 \mathrm{~m}$ with monitor not powered; $n=6$ ).

These results suggest that mice can learn to operate VR engines that render three-dimensional environments, and that vivid landmark cues are critical for this behavior. We next asked whether visual cues are sufficient for navigation by conducting probe trials without rewards. As illustrated in the example position plots from one animal operating a vivid VR environment (Fig. 4A), training sessions dramatically improve their ability to alternate between reward zone locations in the absence of active water rewards. As with the training sessions, vivid landmark cues were necessary for improving performance in probe trials, and mice operating bland tracks showed little change in their performance over the two probe trials (Fig. 4B). Training significantly increased avatar mean velocity on probe trials in both vivid and bland tracks (Fig. 4C; vivid track $P<0.005, n=10$; bland track $P<0.02, n=6$; paired $t$-test). However, only mice operating vivid virtual tracks increased their frequency of midzone crossings in probe trials (Fig. $4 \mathrm{D})(P<0.001)$ and the number of virtual rewards received (reward frequency had the water solenoid not been disabled; $P<$ 0.01). Neither performance metric (midzone crossings and virtual reward frequency) was significantly different between probe trials for mice operating bland virtual tracks $(P>0.05$; paired $t$-test).

We measured the time the avatar spent in different track zones (see Fig. 5A) in probe trials before and after training. The avatar "dwell time" was selectively increased in track zones centered on reward zones (Fig. 5B) $(P<0.02$; paired $t$-test), while the percentages of time spent in the middle of the track and in zones beyond the reward zone were not affected by training $(P>0.05)$. Mice operating bland virtual tracks without vivid landmarks did not increase the percentage of time the avatar spent in the previously rewarded zones $(P>0.05)$ (Fig. 5C).

Head-fixed mice developed stereotyped methods of reversing avatar direction between reward locations during training. By Day 3 of the training period, mice reversed avatar direction without always contacting the end of the track. Figure $6 \mathrm{~A}_{1}$ shows the average of all Day 3 trajectories for 10 mice aligned by reward times. We analyzed the change in avatar view angle in a 30-sec window 
A

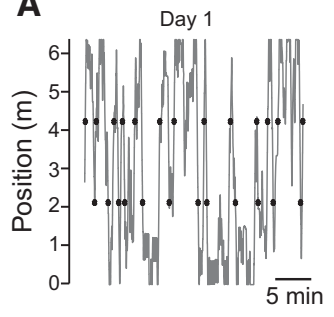

Day 2

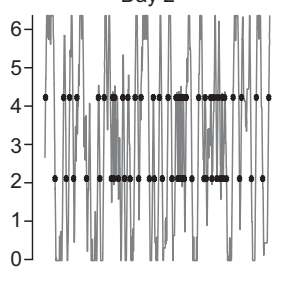

Day 3

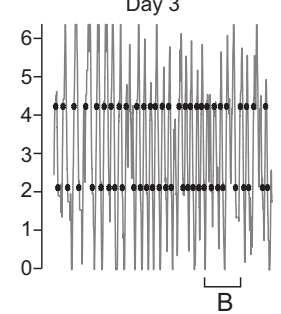

B

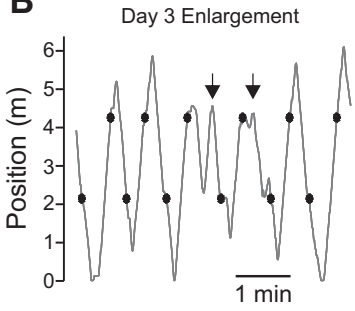

Figure 2. Performance during spatial memory training. $(A)$ Example plots of avatar position along a 6.4-m linear virtual track for one mouse over three training days. Circles indicate water reward times. $(B)$ Enlargement of 5-min position plot indicated by bracket in the right panel in $A$. Arrows indicate re-entry into reward zones.

before the avatar reached the reward zone. In these reverse correlation plots of view angle (Fig. $6 \mathrm{~A}_{2}$ ), mice tested on each training day had clear peaks $\sim 15$ sec before reaching the reward zone. The peak of the average reward-triggered view angle change increased with training, and the mean turning response was significantly increased on Day 3 (Fig. 6B) $(P<0.02)$, suggesting that mice made increasingly larger anticipatory motions using the spherical treadmill with training. It is unlikely that mice improved by learning to turn following a constant delay from the last reward, since reverse correlation plots aligned to the actual reward time plus the mean interval between rewards did not show obvious peaks associated with turning (gray plot in Fig. 6A2, B). Contacting the walls at the ends of the virtual track did not appear to impact performance, as there was no correlation between the percentage of time spent against the end walls and view-angle peak amplitude $\left(R^{2}=-0.003 ; P>0.05\right)$. Instead, training appeared to improve the coordination of their movements on the spherical treadmill when visual landmark cues were present in the vivid track.

Finally, we asked whether turning behavior during training sessions predicted performance on the spatial task when water rewards were disabled. Figure 6C illustrates the correlation between peak view angle modulation on training Day 3 and performance on the second probe trial. These two measures were strongly correlated $\left(R^{2}=0.60 ; P<0.02\right)$ with animals that made the most stereotyped turns (largest average view angle change) between reward locations, displaying better spatial learning as measured by the frequency of virtual rewards in the second probe trial. While the increased stereotypy we observe may reflect the development of more efficient turning behaviors with learning, mice also displayed other behaviors during probe trials, including sitting and circling, which also contribute to dwell time measurements. We found no statistically significant reduction in probe trial performance between the five mice whose avatars frequently contacted the track end walls, which presumably disrupts navigation strategies based on dead reckoning, and the five mice whose avatars spent less time in contact with the end walls $(P>0.05)$. Coordination between treadmill and avatar motion, essential for navigation based on conventional path integration, was disrupted within $6.93 \pm 3.2 \mathrm{sec}(n=10)$ in the final probe trial (5-min duration) after the avatar contacted a wall within the virtual hallway in the vivid track. These results suggest that rodents can navigate between specific locations in virtual environments using solely visual landmark cues.

\section{Discussion}

In this study we demonstrate spatial navigation in head-fixed mice operating a spherical treadmill connected to a VR system. Mice used visual landmark cues to improve their performance in moving an avatar between two water reward locations on a virtual track; control mice operating a bland track lacking landmark cues failed to increase reward frequency during training, as did mice that received no visual feedback. Training with visual landmark cues also improved navigation ability assayed with position-based metrics (virtual reward frequency and frequency of midzone crossings) and by selective increases in the percentage of time spent near the reward zone. The selective increase we find in the dwell time within the previously rewarded zones during probe trials in VR environments is reminiscent of the increase in time spent in the tank quadrant that contained the platform in probe trials in the classic Morris water maze test following spatial learning (Morris 1981). Together, these results demonstrate that head-fixed mice can learn to navigate between spatial locations within a VR environment using only visual cues.

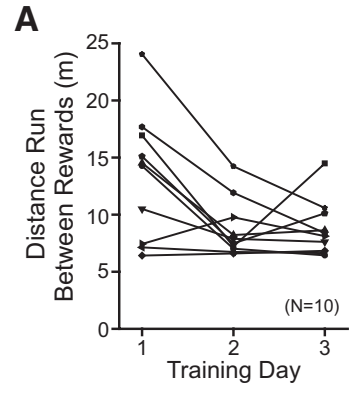

C

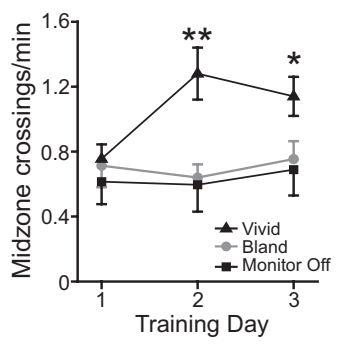

B

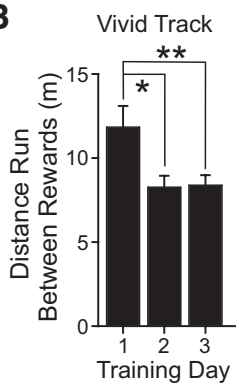

D

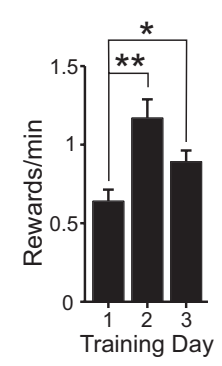

Figure 3. Training improves performance on virtual linear track mazes. $(A)$ Plots of mean distance between rewards vs. training day for animals operating the vivid bidirectional track. (B) Summary plot of mean distance between rewards on each training day for animals operating the vivid (black bars on left) and bland (gray bars on right) tracks. (**) $P<0.01$, $\left({ }^{*}\right) P<0.05$. (C) Plot of the midzone (middle $20 \%$ of track length) crossing frequency in mice controlling vivid and bland virtual environments (top and middle symbols, respectively) and mice operating the VR engine with no visual feedback (Monitor Off, black/bottom symbols). (**) $P<0.01$, (*) $P<0.02$. (D) Plot of frequency of water rewards in each training day for mice operating the vivid virtual track. (**) $P<$ $0.01,\left({ }^{*}\right) P<0.02$. 

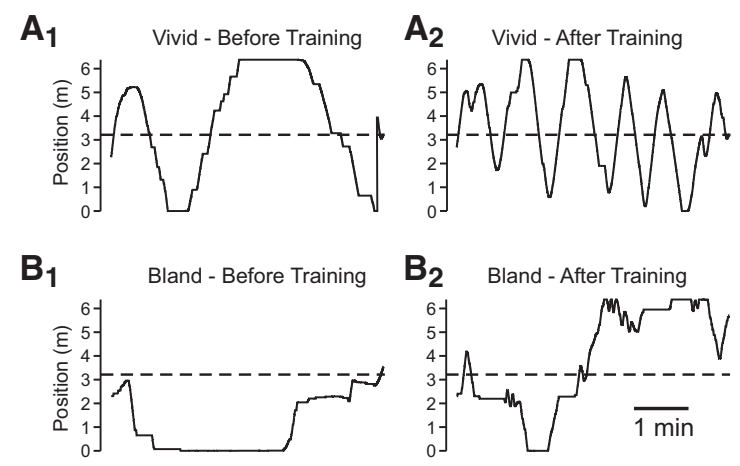

C
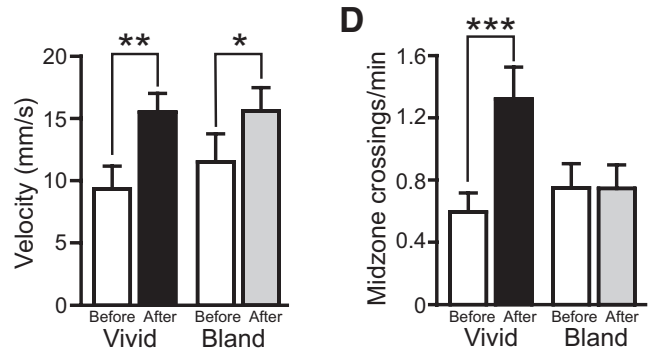

Figure 4. Navigation in virtual environments in the absence of water rewards. (A) Example avatar position plots from probe trials before and after training with a vivid virtual track. (B) Example position plots from probe trials before and after training with a bland virtual track. (C) Plot of mean avatar forward velocity during probe trials before (white bars) and after (black) training in vivid environments and before (white) and after (gray) training with bland virtual tracks. (**) $P<0.002,\left(^{*}\right) P<$ 0.02 . (D) Plot of midzone crossing frequency in the same vivid and bland track groups shown in $C .\left({ }^{* * *}\right) P<0.001$.

While our results are consistent with previous studies that manipulated the visual environment surrounding freely moving rodents (Tanila et al. 1997; Knierim et al. 1998), to our knowledge, no previous study demonstrated spatial navigation when only a single sensory modality conveys reliable information (vision in our study) in nonlesioned rodents. Multiple sensory systems are typically engaged by mobile rodents (e.g., vestibular, olfactory, proprioceptive, somatosensory), all of which can contribute to internal spatial maps. Indeed, Smith et al. (2010) suggested that vestibular input plays a central role in spatial learning-a sensory input that is temporarily blocked while mice are head-fixed. Damage to the vestibular end organs impairs spatial learning abilities in rodents (Baek et al. 2010) and humans (Brandt et al. 2005). Interpretation of these studies is potentially complicated by indirect effects following vestibular damage, including atrophy of other, nonvestibular brain regions (Brandt et al. 2005). Because of the complications in interpreting vestibular lesion studies, it remains difficult to determine whether visually guided spatial learning requires normal vestibular input.

This virtual reality system is uniquely able to isolate the contribution of visual information to navigation, since vestibular input related to head direction is eliminated, and repeated contact with walls within the virtual environment would be expected to rapidly disrupt navigation strategies based on dead reckoning. Similarly, neither somatosensory nor olfactory cues associated with the spherical treadmill have a consistent relationship to reward locations within the virtual environment, aside from the water reward. Previous work addressed multisensory modality integration during spatial navigation by presenting conflicting sensory cues, and found that rodents can improve task performance by prioritizing one sensory modality (Knierim et al.
1998). Our results are consistent with these findings, since nonvisual input is either distorted or eliminated in head-fixed mice operating within a VR environment.

While other studies have used neurophysiological recordings to demonstrate place field activity in rodents (Harvey et al. 2009) and humans (Ekstrom et al. 2003) operating within VR environments, none of these studies assayed spatial ability directly using behavioral tests. Several lines of evidence demonstrate that development of place cell activity does not necessarily imply that spatial learning has occurred. First, the time course of place field development and spatial memory are different. Spatially selective firing patterns in hippocampal units develop rapidly upon entering a new environment (Frank et al. 2004), and also change rapidly when specific visual cues are changed, with plasticity occurring within minutes (Knierim et al. 1995). Even a single pass through a novel environment (a newly open arm on a radial maze) can create new, stable place fields (Frank et al. 2004). However, that single exposure presumably does not result in new long-term spatial memories, since rodents continue to treat the new arm as novel upon subsequent testing. In contrast, demonstrable spatial memory develops over repeated exposures to an environment and is typically assayed over time periods of hours to days (e.g., Blum et al. 1999; for review, see Morris 1984). Second, place cell function and spatial learning can be dissociated experimentally. Blockade of NMDA receptors prevents formation of new spatial memories without preventing the formation of new place fields (Ungerer et al. 1991; Kentros et al. 1998). Chemical lesions of the dentate gyrus also block spatial learning without preventing spatially selective hippocampal unit firing patterns (McNaughton et al. 1989). Third, the interpretation of spatially selective firing patterns is complicated by the polysensory nature of hippocampal neurons. Nonvisual stimuli, for example, often can activate "spatially selective" hippocampal units, regardless of the physical location of the animal (Wood et al. 1999; Save et al. 2000) (for review, see Eichenbaum et al. 1999). Specific tests are required to determine whether place-specific firing behaviors actually reflect
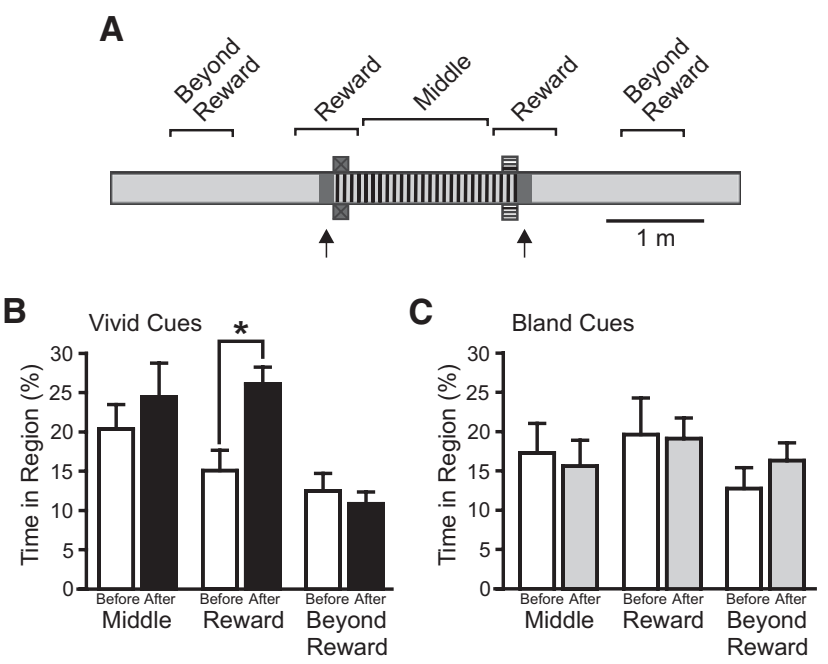

Figure 5. Training selectively increases time spent near reward locations. (A) Diagram of virtual linear track and analysis compartments. (B) Plot of percentage of time spent near reward zone (Reward, $10 \%$ of track length centered on each reward trigger zone), in the middle $20 \%$ of the track (Middle) and in two 10\% zones beyond each reward zone (Beyond Reward, $0.64 \mathrm{~m}$ past the reward zone) in experiments with vivid landmarks. ( $\left.{ }^{*}\right) P<0.02$. (C) Plot of percentage of time spent in track zones in experiments with the bland track. 
$A_{1}$
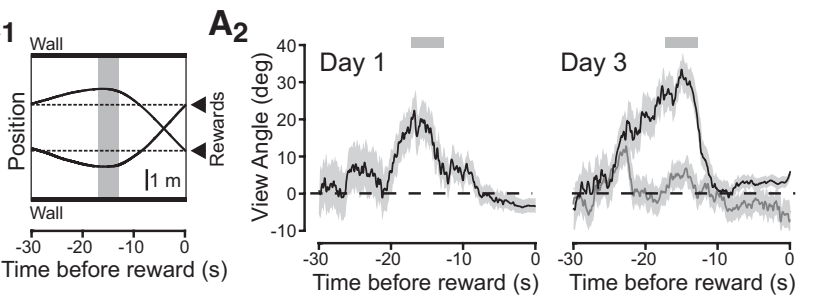

B

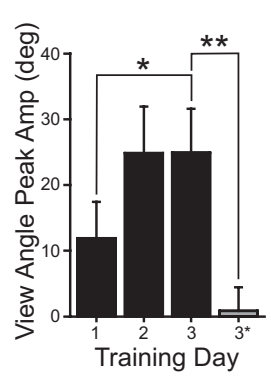

C

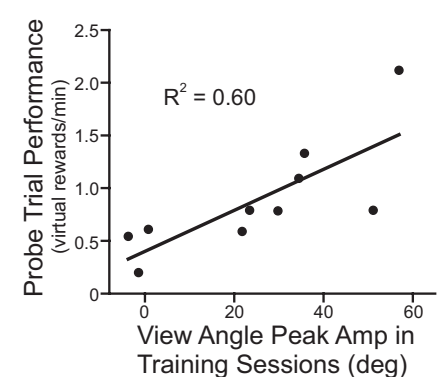

Figure 6. Stereotyped turning movements predict probe trial performance. $\left(A_{1}\right)$ Avatar position immediately before rewards on training Day 3. Average trajectories synchronized on reward times and plotted separately for lower (bottom line) and upper (top line) reward zones. Avatars initially continue beyond the previous reward zone before changing direction at $\sim 15 \mathrm{sec}$ before reaching the next reward zone. $\left(A_{2}\right)$ Avatar view angle position before reaching reward trigger location from trials in training Days 1 and 3. Average view angle computed as deviation in degrees from direct (track midline) approach to the target ( $n=368$ and 534 rewards, respectively; $n=10$ animals tested on vivid track). Lower, gray plot on right panel is a reverse correlation of training Day 3 based on a regular interval (the mean interval between actual rewards received). Shaded regions represent SEM. (B) Plot of mean view angle in analysis window (horizontal top bar in $A_{2}$ ) for all 10 animals on training Days 1 3 and on the time-shifted correlation Day 3 control plot $\left(3^{*}\right)$. (*) $P<$ 0.02 , (**) $P<0.01$. (C) Plot of the relationship between the increase in avatar view angle prior to reaching reward (as in $B$ ) in training sessions on Day 3, and performance on the second probe trial (following training) for the 10 mice in the vivid group.

internal spatial maps or, instead, reflect sensory cues associated with specific locations. When this analysis is performed, often only a minority of hippocampal units have place fields that are solely spatially selective and do not also respond to sensory input associated with that location (Ekstrom et al. 2003). Spatially selective firing patterns also typically do not imply an absence of responses outside of the preferred place field. Rather, place cell activity reflects a bias toward stronger responses in one spatial region compared with other regions.

Our results also demonstrate a strong correlation between motor stereotypy (consistent modulation of avatar view angle during turning) (Fig. 6A,B) and performance on a spatial navigation test. This analysis was feasible because rewards were located near the middle third of the track, compared with related VR studies with rewards located near track boundaries (Harvey et al. 2009; Dombeck et al. 2010). This type of correlation has been observed in previous spatial memory studies in freely moving pigeons (Schwartz 1982), though another report (Janis et al. 1994) found that behavioral stereotypy was negatively correlated with spatial learning in rodents. The behavioral task used in our experiments almost certainly involves visuo-motor coordination to operate the spherical treadmill to control avatar movement, which could account for the increasingly stereotyped motor behaviors we find during the final stage of training (Olton 1979). In principle, the increased stereotypy may reflect a switch from navigation strategies based on spatial memory to serial motor strategies as the mice become familiar with the environment (Stamps 1995). Future studies will be required to determine whether the learning we demonstrate reflects primarily improved visuo-motor coordination or the formation of new spatial memories, and which behaviors contribute to the dwell time changes following training.

While our study used VR to control the salience of different types of sensory input, we did not address how the experimental animals made use of the visual information presented to perform the spatial task. It is possible that mice interpret the optic flow patterns with appropriate perspective rendered by the VR system as a three-dimensional environment. However, our study cannot eliminate the possibility that mice learn to navigate through the track simply by recognizing the consistent relationship between landmark renderings and reward locations. A significant body of research has dissociated between cue-guided spatial navigation and navigation guided by recall of spatial memory, and has provided evidence for parallel spatial processing within different brain structures, as well as within the hippocampus (Morris 1981; McDonald and White 1994; Gothard et al. 1996). Previous work has suggested that rodents prefer to rely on visual cues over nonvisual cues when possible or when other systems are providing less-reliable information, as is seen in the reliance on visual landmarks in vestibular-lesioned rats (Stackman and Herbert 2002). In the Morris water maze, spatial memory is classically tested in probe trials with identical conditions to training but without the goal, the escape platform. In our study, we removed the goal, the water reward, and found the mice continued to navigate the avatar to the virtual locations where the goal had been. Our results are consistent with both the development of spatial memories for reward locations during training and improved cue-guided navigation abilities. Future studies in which the normal perspectivedriven VR rendering is distorted will be necessary to dissect the most salient visual cues for spatial navigation. The large difference in performance that we found between animals operating the vivid and bland VR environments indicates only that visual cues are necessary for spatial navigation in this task.

We believe this study represents the first demonstration of spatial learning in head-fixed rodents operating in a virtual environment. Hölscher et al. (2005) demonstrated spatial navigation in VR in rats suspended with a halter over a spherical treadmill surrounded by a toroidal projection screen. Since the halter only restricted movement of experimental animals to a region near the apex of the spherical treadmill, this study could not distinguish the relative importance of visual and vestibular input in promoting spatial navigation. In contrast, vestibular input was eliminated in both our study and in the recent studies from Tank and colleagues (Harvey et al. 2009; Dombeck et al. 2010) by headfixation. The observation that place fields can be recorded in hippocampal neurons using electrophysiological (Harvey et al. 2009) and imaging (Dombeck et al. 2010) methods is consistent with the hypothesis that head-fixed rodents can form spatial memories in virtual environments, though neither study tested spatial memory directly. The findings in virtual environments are consistent with those of real world environments in which place cell firing has been found to be influenced by the direction of motion, idiothetic input, and external sensory cues (Gothard et al. 1996; Redish et al. 2000). Place fields recorded in linear hallway tasks appear to respond more strongly to path integrative information than to external sensory information, although the fields were found to shift toward a dependence on external sensory cues if they were more reliable (Gothard et al. 1996). In our study, only visual cues and somatosensory self-motion information were available for navigation, and the walls within the virtual environment rapidly disrupt path integration strategies. Therefore, path integration is unlikely to guide spatial behavior in our task, suggesting that navigation through the virtual hallway relies on visual cues alone. 
The ability to promote spatial learning using only salient visual cues should facilitate future work aimed at defining the cellular changes associated with spatial memory. This experiment is not easily tractable in freely moving rodents since, most likely, neural substrates of spatial learning are polysensory brain regions, where it is difficult to associate the relevant sensory inputs with plasticity-related cellular modifications. Our results suggest that by using VR with head-fixed rodents, it may be possible to link neural responses to specific visual stimuli with cellular changes associated with learning.

\section{Materials and Methods}

\section{Surgical procedures}

Wild-type BL6 mice $6-8$ wk old $(n=22 ; 16$ male and six female; Harlan Laboratories) were trained to run to water-reward locations on a virtual track. All surgical and training procedures were approved by the CWRU IACUC Committee. Mice initially underwent a surgical procedure (ketamine $100 \mathrm{mg} / \mathrm{kg}$, xylazine $10 \mathrm{mg} /$ $\mathrm{kg}$ i.p. anesthesia) $3 \mathrm{~d}$ before training to mount a titanium small plate $(9 \times 25 \mathrm{~mm})$ with two bolt holes to the dorsal surface of the skull used to fix head position using Metabond (Parkell) adhesive. Two days following the head plate surgery, mice were water restricted and maintained at $\sim 85 \%$ of their presurgery body weight throughout the study. Two mice were eliminated from the study when their head plates detached or because of illness.

\section{Apparatus}

Mice were positioned near the apex of a levitated 20.3-cm-diameter Styrofoam spherical treadmill (Fig. 1A; similar to Harvey et al. 2009) using two 4-40 metal bolts that connected the head plate to an air table (RS2000, Newport) via stainless-steel posts. A 16-gauge sip tube (FNC-16-3; Kent Scientific) was positioned 2-4 mm in front of the mouse to deliver $10-\mu \mathrm{L}$ water rewards. Mice could run on the treadmill to operate the VR system described below (Fig. 1A). Mice could also sit, groom, and grasp the sip tube while the treadmill was levitated. All experiments were conducted within a blackout box with the LCD monitor that rendered the VR output as the only source of illumination.

\section{Training protocol}

Mice were trained to run through a virtual hallway in three phases. In the initial phase (four sessions, $30 \mathrm{~min}$ each, two sessions per day) (Fig. 1B), mice were conditioned to the apparatus without visual feedback by providing water rewards in proportion to the backward velocity of the treadmill (rewards became more frequent as the mouse walked forward more rapidly). Following the four conditioning sessions, mice were trained to run through a virtual hallway in a unidirectional task (six sessions, 30 min each, two sessions per day, see below for details) with visual feedback generated by the VR system. The VR avatar was teleported back to the track origin after each water reward. In the final phase, mice were trained to run back and forth between two water rewards located in a virtual hallway. Mice were assisted with turns following water rewards in the first 30-min bidirectional training session, as in the Harvey et al. (2009) study. Mice were unassisted in the remaining seven bidirectional sessions (each $30 \mathrm{~min}$, two sessions per day). The present study is based on the results acquired during the final six bidirectional sessions and in probe trials (5 min each) without water rewards, conducted immediately before and after the bidirectional training phase.

\section{Behavioral tasks}

We used standard Unity built-in functions and short macros to construct virtual 6.4-m unidimensional hallway tracks. A simple unidirectional track was used for the second phase of training with a single reward trigger located at $2.1 \mathrm{~m}$ from the track origin. In most experiments (those with "vivid" tracks), hallway floors and walls were decorated with varying patterns; reward zones were denoted with green floors and blue patterned walls. We also constructed two towers, which stood next to the track near reward locations (see Supplemental Video). Mice have dichromatic vision with peak cone sensitivities near green and UV wavelengths (Jacobs et al. 2004; Wang et al. 2011). While the reward floor area color is detected well by mid-wavelength opsins (Applebury et al. 2000), we did not directly test whether mice could distinguish among the other colors rendered. Mice $(n=20)$ were trained to run through virtual unidirectional hallways by initially placing the VR avatar at the track origin and delivering $10-\mu \mathrm{L}$ water rewards when the avatar reached the trigger zone. The VR avatar was then teleported back to the track origin and the task repeated (30-min sessions, two sessions per day). Avatar view angle modulation sensitivity (used to turn right and left) was initially zero, and then was increased during each unidirectional track training session until it reached the magnitude used in bidirectional tracks. Mice were divided randomly in three groups. One group $(n=10)$ operated the vivid visual cues in both unidirectional and bidirectional tracks. A second group $(n=6)$ operated a "bland" track with no hallway decorations or towers near the reward zone. The geometry and reward locations in "vivid" and "bland" tracks were identical. A final group $(n=6)$ operated the VR system without any visual feedback (LCD monitor was not powered) during unidirectional and bidirectional phases.

In the final track configuration ("bidirectional"; eight sessions of 30-min each), mice were placed in a virtual hallway with two water-reward locations (at 2.1 and $4.2 \mathrm{~m}$ ); mice only received water rewards when the avatar alternated between both reward sites. By placing reward zones away from the track ends, we were able to assess the development of their ability to reverse the avatar direction by rotating the treadmill along its equator to modulate view angle. We used two variants of the same bidirectional track: a "vivid" track that had hallway and reward zone decorations similar to those presented in the unidirectional track, and a "bland" bidirectional track that lacked visual landmarks. Avatars were not teleported in the bidirectional task. Instead, the avatar was placed at the track origin at the beginning of each session, and only moved in response to treadmill motion.

Development of spatial navigation skill was tested in probe trials (5-min duration) conducted immediately before and after the eight-session bidirectional training block. The same track configuration ("vivid" or "bland") the mice experienced during bidirectional training was used during both probe trials. Water rewards were disabled in probe trials to assess the relative contributions of visual cues and active rewards to spatial navigation ability.

\section{Virtual reality system}

Rotations along two axes of the treadmill were detected using an optical mouse (MX518, Logitech) positioned next to the treadmill and connected to a Windows netbook computer (Eee1005HA, Asus). In the initial phase of training ("instrument conditioning") (Fig. 1B), a custom Visual Basic.NET (VB.NET, Microsoft) program activated a solenoid valve to deliver $10-\mu \mathrm{L}$ water rewards when forward rotational velocity exceeded a threshold that was increased with each session.

In the second ("unidirectional track") and third ("bidirectional track") phases of training, mice were exposed to a continuous VR display generated in real time by the Unity video game engine (version 2.6.1; Unity Technologies) running on a second Windows computer. Unity output was rendered on a single widescreen LCD display (2433BW, Samsung) positioned $30 \mathrm{~cm}$ in front of the mouse. A second custom netbook VB.NET program converted treadmill rotational velocities into analog $0-5 \mathrm{~V}$ signals using a USB multifunction device (U3, Labjack). These analog voltages were applied to a U-HID-Nano input device (Ultimarc) connected to the VR computer that emulated a USB gaming joystick. This system enabled mice to operate the video game engine (controlling both forward/backward movement and avatar view angle) without requiring any modifications to the Unity program. A third VB.NET program operated the solenoid valve that controlled water rewards and was called by a Unity script when the mouse 
entered a reward trigger zone. Treadmill rotations generated by head-fixed mice running straight moved the VR avatar forward (Fig. $1 \mathrm{~A}_{2}$ ); rotation along the treadmill equator modulated view angle. Mice could reverse direction on the bidirectional track by modulating the avatar view angle to turn left or right. Avatar motion stopped if the input vector generated by the mouse-operated treadmill was perpendicular to the hallway wall contacted. Contact with hallway walls at oblique angles only blocked one component of the input motion vector.

\section{Statistical analysis}

Avatar position, view angle, and reward status was logged during each video frame update (every $10-30 \mathrm{msec}$ ). To simplify data analysis, results from the last six bidirectional training sessions were combined into three groups: Day 1 (bidirectional sessions 3 and 4), Day 2 (sessions 5 and 6), and Day 3 (sessions 7 and 8). (We began our analysis with session 3 since mice were prompted to turn by the experimenter during the initial phase of bidirectional training; all results analyzed in Days 1-3 were from unassisted sessions.) Reward-time averages were computed from interpolated (10-msec intervals) avatar view angles to compensate for variable frame rendering rates. Reward-triggered averages of view angle were smoothed using a moving Savitzky-Golay filter (310-msec duration, 3rd order). Avatar turning responses (Fig. $6 \mathrm{~A}_{2}$ ) were calculated by averaging the magnitude of view angle deviation from either 90 or 270 degrees, depending on the next active reward zone. Data analysis was performed in Matlab $2010 \mathrm{~b}$ (Mathworks); Origin 8.1 (OriginLab) was used to conduct statistical tests. Results are presented as mean \pm SEM, and statistical significance was assessed using Student's t-test.

\section{Acknowledgments}

We thank Drs. R. Galan, J. Knierim, D. Wesson, and P. Larimer for helpful discussions and critical comments on this manuscript. This work was supported by NIH grants DC04285 and DC09948. I.A.Y. was supported by NIH training grant T32-AG00271.

\section{References}

Applebury ML, Antoch MP, Baxter LC, Chun LL, Falk JD, Farhangfar F, Kage K, Krzystolik MG, Lyass LA, Robbins JT. 2000. The murine cone photoreceptor: A single cone type expresses both $\mathrm{S}$ and $\mathrm{M}$ opsins with retinal spatial patterning. Neuron 27: 513-523.

Baek JH, Zheng Y, Darlington CL, Smith PF. 2010. Evidence that spatial memory deficits following bilateral vestibular deafferentation in rats are probably permanent. Neurobiol Learn Mem 94: 402-413.

Blum S, Moore AN, Adams F, Dash PK. 1999. A mitogen-activated protein kinase cascade in the CA1/CA2 subfield of the dorsal hippocampus is essential for long-term spatial memory. J Neurosci 19: 3535-3544.

Brandt T, Schautzer F, Hamilton DA, Brüning R, Markowitsch HJ, Kalla R, Darlington C, Smith P, Strupp M. 2005. Vestibular loss causes hippocampal atrophy and impaired spatial memory in humans. Brain 128: $2732-2741$.

Brown MF, Yang SY, DiGian KA. 2002. No evidence for overshadowing or facilitation of spatial pattern learning by visual cues. Anim Learn Behav 30: $363-375$.

Dombeck DA, Harvey CD, Tian L, Looger LL, Tank DW. 2010. Functional imaging of hippocampal place cells at cellular resolution during virtual navigation. Nat Neurosci 13: 1433-1440.

Eichenbaum H, Dudchenko P, Wood E, Shapiro M, Tanila H. 1999. The hippocampus, memory and place cells: Is it spatial memory or memory for space? Neuron 23: 209-226.

Ekstrom AD, Kahana MJ, Caplan JB, Fields TA, Isham EA, Newman EL, Fried I. 2003. Cellular networks underlying human spatial navigation. Nature 425: $184-187$.

Etienne AS, Jeffery KJ. 2004. Path integration in mammals. Hippocampus 14: $180-192$.

Frank LM, Stanley GB, Brown EN. 2004. Hippocampal plasticity across multiple days of exposure to novel environments. J Neurosci 24: $7681-7689$.

Gothard KM, Skaggs WE, McNaughton BL. 1996. Dynamics of mismatch correction in the hippocampal ensemble code for space: Interaction between path integration and environmental cues. J Neurosci 16: 8027-8040.

Harvey CD, Collman F, Dombeck DA, Tank DW. 2009. Intracellular dynamics of hippocampal place cells during virtual navigation. Nature 461: $941-946$.

Hölscher C, Schnee A, Dahmen H, Setia L, Mallot H. 2005. Rats are able to navigate in virtual environments. J Exp Biol 208: 561-569.

Jacobs GH, Williams GA, Fenwick JA. 2004. Influence of cone pigment coexpression on spectral sensitivity and color vision in the mouse. Vision Res 44: $1615-1622$.

Janis LS, Bishop TW, Dunbar GL. 1994. Medial septal lesions in rats produce permanent deficits for strategy selection in a spatial memory task. Behav Neurosci 108: 892-898.

Janus C. 2004. Search strategies used by APP transgenic mice during navigation in the Morris water maze. Learn Mem 11: 337-346.

Kentros C, Hargreaves E, Hawkins RD, Kandel ER, Shapiro M, Muller RV. 1998. Abolition of long-term stability of new hippocampal place cell maps by NMDA receptor blockade. Science 280: 2121-2126.

Knierim JJ, Kudrimoti HS, McNaughton BL. 1995. Place cells, head direction cells, and the learning of landmark stability. J Neurosci 15: $1648-1659$

Knierim JJ, Kudrimoti HS, McNaughton BL. 1998. Interactions between idiothetic cues and external landmarks in the control of place cells and head direction cells. J Neurophysiol 80: 425-446.

Lever C, Wills T, Cacucci F, Burgess N, O'Keefe J. 2002. Long-term plasticity in hippocampal place-cell representation of environmental geometry. Nature 416: $90-94$

McDonald RJ, White NM. 1994. Parallel information processing in the water maze: Evidence for independent memory systems involving dorsal striatum and hippocampus. Behav Neural Biol 61: 260-270.

McNaughton BL, Barnes CA, Meltzer J, Sutherland RJ. 1989. Hippocampal granule cells are necessary for normal spatial learning but not for spatially-selective pyramidal cell discharge. Exp Brain Res 76: 485-496.

Morris R. 1981. Spatial localization does not require the presence of local cues. Learn Motiv 12: 239-260.

Morris R. 1984. Developments of a water-maze procedure for studying spatial learning in the rat. J Neurosci Methods 11: 47-60.

Olton DS. 1979. Mazes, maps, and memory. Am Psychol 34: 583-596.

Redish AD, McNaughton BL, Barnes CA. 2000. Place cell firing shows an inertia-like process. Neurocomputing 32-33: 235-241.

Roberts AD, Pearce JM. 1999. Blocking in the Morris swimming pool. J Exp Psychol Anim Behav Process 25: 225-235.

Save E, Moghaddam M. 1996. Effects of lesions of the associative parietal cortex on the acquisition and use of spatial memory in egocentric and allocentric navigation tasks in the rat. Behav Neurosci 110: 74-85.

Save E, Nerad L, Poucet B. 2000. Contribution of multiple sensory information to place field stability in hippocampal place cells. Hippocampus 10: $64-76$.

Schwartz B. 1982. Failure to produce response variability with reinforcement. J Exp Anal Behav 37: 171-181.

Smith PL, Darlington Cl, Zheng Y. 2010. Move it or lose it-Is stimulation of the vestibular system necessary for normal spatial memory? Hippocampus 20: $36-43$.

Stackman RW, Herbert AM. 2002. Rats with lesions of the vestibular system require a visual landmark for spatial navigation. Behav Brain Res 128: $27-40$.

Stamps J. 1995. Motor learning and the value of familiar space. Am Nat 146: $41-58$.

Tanila H, Shapiro ML, Eichenbaum H. 1997. Discordance of spatial representation in ensembles of hippocampal place cells. Hippocampus 7: $613-623$.

Ungerer A, Mathis C, Me'lan C, De Barry J. 1991. The NMDA antagonists, CPP and $\gamma$-l-glutamyl-l-aspartate, selectively block post-training improvement in a Y-maze avoidance learning task. Brain Res 549: 59-65.

Wang YV, Weick M, Demb JB. 2011. Spectral and temporal sensitivity of cone-mediated responses in mouse retinal ganglion cells. J Neurosci 31: 7670-7681.

Wilson MA, McNaughton BL. 1993. Dynamics of the hippocampal ensemble code for space. Science 261: 1055-1058.

Wood ER, Dudchenko PA, Eichenbaum H. 1999. The global record of memory in hippocampal neuronal activity. Nature 397: 613-616.

Zheng Y, Goddard M, Darlington CL, Smith PF. 2009. Long-term deficits on a foraging task after bilateral vestibular deafferentation in rats. Hippocampus 19: 480-486.

Received July 15, 2011; accepted in revised form December 27, 2011. 


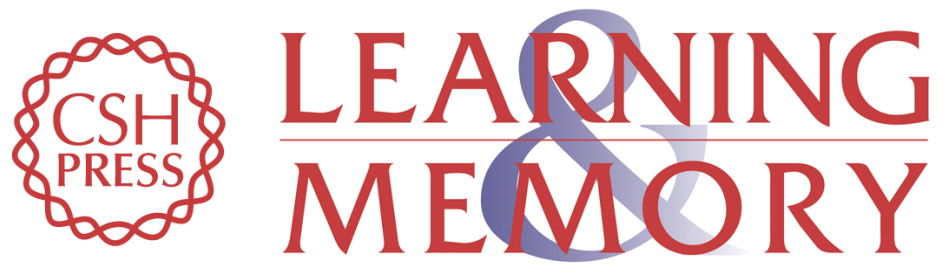

\section{Visual landmarks facilitate rodent spatial navigation in virtual reality environments}

Isaac A. Youngstrom and Ben W. Strowbridge

Learn. Mem. 2012, 19:

Access the most recent version at doi:10.1101//m.023523.111

Supplemental
Material http://learnmem.cshlp.org/content/suppl/2012/02/09/19.3.84.DC1

References This article cites 39 articles, 9 of which can be accessed free at: http://learnmem.cshlp.org/content/19/3/84.full.html\#ref-list-1

License

Email Alerting Receive free email alerts when new articles cite this article - sign up in the box at the Service top right corner of the article or click here. 